\title{
Metal Casting Energy Efficient Metrics for Material Selection of Automotive Parts
}

\author{
Emanuele Pagone, Michail Papanikolaou, Konstantinos Salonitis, Mark Jolly
}

\author{
Sustainable Manufacturing Systems Centre, School of Aerospace, Transport and Manufactur- \\ ing, Cranfield University, Cranfield, MK43 0AL, United Kingdom \\ e.pagonedcranfield.ac.uk
}

\begin{abstract}
The automotive sector is one of the main end-use markets for metal casting worldwide. The strong competitive pressure typical of this industry have been influenced in the recent years by sustainability as a new factor promoted by legislation, increased societal awareness of relevant instances and resource scarcity. Energy efficiency, although only a part of sustainability, is important for the metal casting practice because of its nature of large consumer of energy per unit product. Therefore, the effective use of appropriate energy efficient metrics in foundries is of great interest. In this work, a set of indicators developed by the authors (and derived by traditional metrics) to analyse the energy performance of foundries will be used to compare high pressure die casting processes producing car transfer cases with different suitable materials. On the basis of this analysis, it will be shown that the most energy efficient material can be identified whereas the traditional metrics cannot detect such opportunity.
\end{abstract}

Keywords: Casting, Energy Efficiency, Decision Making.

\section{Introduction}

The automotive industry is one of the main business sectors for metal shape casting: e.g., it was $35 \%$ of the USA end-use markets in 2004 [1], about $43 \%$ of the global aluminium casting market in 2011 [2] and 32\% of the Indian market in 2017 [3]. Although this industry has always been subjected to strong competition, in the last years automotive manufacturers have been at the centre of environmental concerns, especially with regards to passenger light vehicles. Considering that, as shown, metal casting is an integral part of the automotive industry supply-chain, it contributes significantly in responding to environmental concerns or, more generally, to instances of sustainability that are becoming more and more important.

For these reasons, after a general overview of decision making metrics in manufacturing systems, energy efficient metrics for the evaluation and benchmarking of foundries will be presented. Finally, a case study comparing three suitable materials to produce the same automotive part according to the mentioned energy efficient metrics, will be analysed. 


\section{Decision Making Metrics in Manufacturing Systems}

Manufacturing systems are complex and multi-disciplinary in nature requiring expertise both in technical and management areas. A typical description of their conceptual structure identifies a few main categories [4].

- Processes acting on the materials produced;

- Equipment necessary to practically implement the mentioned processes;

- Material and information flows that connect the workforce to the equipment;

- Interfaces between design and the actual manufacturing of a product.

In particular, material and information flow analyses can be supported by mapping also the energy flows to provide a more thorough description of the manufacturing system, especially when its core activities are characterised by high amounts of energy per unit product. Such "energy intensive" manufacturing processes, like casting, largely benefit from this additional study. A practical example of the advantages derived by this approach is understanding, benchmarking and improving the full chain of metal casting processes [5]. Another approach is combining multi-layer stream mapping with near-real-time monitoring [6] or with eco-efficiency methods $[7,8]$ to create a decision making framework.

Thus, decision making in manufacturing systems emerges as a complex combination of several disciplines aimed at achieving business goals through management and technical means.

A well-established classification of manufacturing metrics identifies four major, interrelated areas where Key Performance Indicators (KPIs) can be categorised. Namely, these mentioned areas are cost, time, quality and flexibility [4]. However, recent significant changes in the context where the modern manufacturing systems operate, together with a cultural evolution of its values, have promoted the expansion of these areas to consider an additional category: sustainability (Fig. 1). This is the ability of mankind to endure in the long-term preserving key economic, environmental and social aspects [9]. An obvious requirement of sustainability is satisfying current needs in a way that weights its long-term implications in the three mentioned areas [10].

Obviously, alternative ways to classify manufacturing metrics are possible. For example, industrial ecology frameworks incorporating the concepts of eco-efficiency and industrial symbiosis, organise the same main concepts presented in a different way [11].

Regardless of their specific classification, the simultaneous minimisation or maximisation of different KPIs is not generally possible and, thus, optimisation studies are necessary to design and operate manufacturing systems $[4,12]$. To achieve this goal, it is of critical importance to quantify precisely attributes also in the cases when indicators are more easily defined qualitatively rather than quantitatively. 


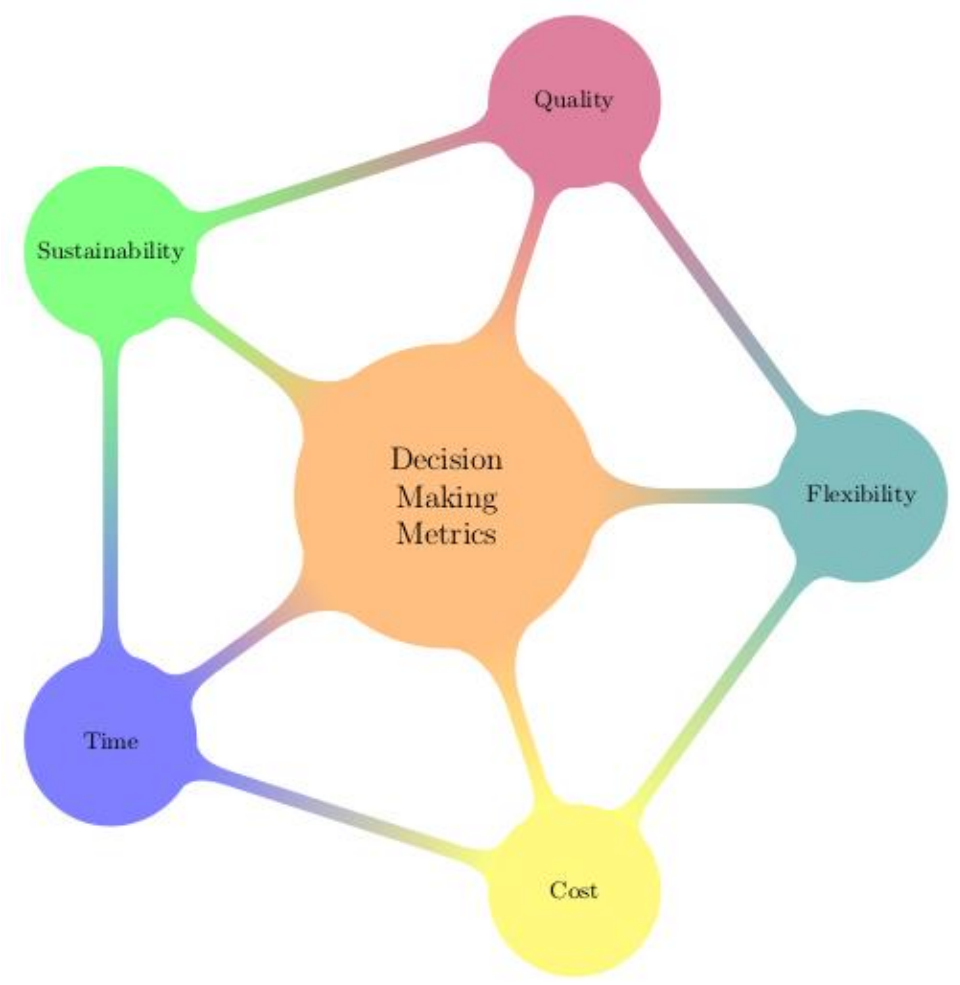

Fig. 1. Decision making metrics can be grouped by five main classes: cost, time, quality, flexibility and sustainability.

\subsection{Conventional Metrics}

Considerable efforts have been dedicated to the study of trade-offs between cost and time required to produce a product. Historically, this has been the first problem in designing and operating manufacturing systems after WWII. In the Seventies, quality characteristics have become equally important and finally, at the end of last century, flexibility has arisen as a critical component to competitiveness caused by changes in the market [4].

Costs quantification in manufacturing is a broad topic that can be generally summarised considering the following areas: materials, energy, labour, training, facilities, equipment, maintenance, overhead (to support manufacturing activities) and capital costs [4].

Time is generally intended as production rate and it is measured in relationship to productivity metrics. The overall production rate efficiency $\eta_{p}$ is the main indicator of the system performance and is calculated considering the theoretical and actual production rate, $P_{t}$ and $P_{a}$ respectively:

$$
\eta_{p}=\frac{P_{a}}{P_{t}}
$$


However, in order to know the value of $P_{t}$ is necessary to consider the productivity metrics of the machines comprising the system and their arrangement. Other useful time-related metrics are the production schedule of the plant as well as its volume of production over a certain amount of time. The productivity of individual machines can be assessed on the basis of reliability considering the following quantities [4]:

- Mean Time Between Failures (MTBF) [13];

- Mean Time To Repair (MTTR) [13];

- failure rates $\lambda$ defined as the number of fails over a specific amount of time;

- the magnitude of reliability $R=e^{-\lambda t}$ (where $t$ is time);

Moreover, lean-thinking related metrics, like lead time, cycle time, work-inprocess and others, are particularly fit to describe productivity both of individual machines and the overall performance [6-8]. Also reliability or availability KPIs can be combined to assess the production rate of the entire plant starting from the values of single machines.

One of the most basic ways to measure quality in a manufacturing system is calculating the fraction of defective products. The associated warranty costs can be useful in performing cost optimisations in a simpler way [4]. However, although these values are relatively easy to obtain and calculate, they do not provide more granular information about the specific processes comprising the system. To analyse the performance with respect to quality more specifically, metrics like the process capability index $C_{p}$ [4] or the quality loss function $L[14]$ are better suited:

$$
\begin{gathered}
C_{p}=\frac{\tau}{6 \sigma} \\
L_{x}=\frac{A}{\tau^{2}}(x-m)^{2}
\end{gathered}
$$

where $\tau$ is the tolerance limit, $x$ is a specific quality characteristic being assessed, $\sigma$ is the standard deviation of $x$ generated by the process, $A$ is the cost to reproduce or repair the part and $m$ is the production target value.

Finally, flexibility can be considered in different ways and includes a large number of cases: changes in machines, processes, products, routing, production volumes, facilities, operations and part types [15]. Buzacott distinguishes between the ability to cope with variable external requests and the efficiency to react against disturbances within the system (mostly related to reliability) [16]. In the first case, flexibility can be represented by the probability to perform a certain operation, if it is within the capabilities of the plant. Another class of methods in this area have the advantage to be linked to economic models and are based on discounted cash flow techniques. These methods aim at quantifying the intangible benefits of flexibility [4]. On the other hand, while assessing the ability to cope against internal disturbances, several metrics can be considered:

- the ratio of set-up time over cycle time that can be applied both at machine and at system level [17]; 
- a mathematical expression of flexibility similar to entropy combining machine reliabilities or availabilities normalised [18];

- a weighted expression benchmarked against a pre-determined set of tasks [19];

- indices at process and system level counting the relevant number of design changes and reconfigurations in a year [20].

However, the calculated value of flexibility metrics that do not take into account external demand (although easier to calculate) does not necessarily represent a measure of the variability actually requested. This disadvantage is partially reduced only in the case of the indicator benchmarked against a set of tasks where tweaking the weights in its expression it is possible to represent the needed flexibility [4]. With the aim to resolve also this issue, Chryssolouris suggests as a "generic measure of flexibility" the Penalty Of Change (POC), an attribute inversely proportional to flexibility:

$$
P O C=P n(x) \operatorname{Pr}(x)
$$

where $x$ is the variable representing a change, $P n$ is the penalty associated with its variation and $P r$ is its probability to occur. The penalty of change should be evaluated in the regards of the product, operations or production rate. The value of POC can be calculated in manufacturing systems where discrete changes are possible performing the sum of all the combinations describing the changes or, in case of continuous change of states, via integration [4].

\subsection{Sustainability Metrics}

Sustainability is traditionally considered according to three interrelated areas: social, environmental (or ecological) and financial (or economic) impact [9]. Salonitis and Stavropoulos have suggested to substitute the class of "cost" in the categorisation of decision making attributes (depicted in Fig. 1) with the economic dimension of sustainability [21,22]. Being a relatively new concept (initially conceived at the beginning of the Nineties of the last century [10]), the development and standardisation of sustainability cannot be considered to have reached full maturity yet. However, a significant amount of research has been dedicated to the development of methods for sustainability appraisal, counting as many as 50 tools in 2013 [23].

Considering the ambitious and comprehensive scope of sustainability, a large number of metrics is often required, making the relevant assessment demanding in terms of time and resources. Furthermore, although some of the tools available do allow a relatively quick evaluation, sometimes they do not cover the full sustainability spectrum across the three areas previously mentioned or do not permit comparisons between different types of industry because the assessment is not general enough. Tools that exemplify these shortcomings are the Sustainable development in the European Union [24] (based on 100 indicators), the IChemE Sustainability Metrics [25] (requiring more than 300 values and it is restricted mostly to the process industry) or the Barometer of Sustainability [26] (suitable for quick assessments, but it considers economic aspects with limited attention). 
Considering the diverse context of the sustainability tools described and the large number of metrics that each methodology adopts, it would be impossible to provide a comprehensive review of such indicators in this work. Hence, just examples of typical sustainable manufacturing variables follow [22].

- Environmental. Quantity of pollutant emitted in terms of: global warming potential (expressed as amount of carbon dioxide equivalent), solids released as process waste or into water sources, acidifying compounds discharged in process water. Waste process heat rejected to process water, specific material and energy consumption per product.

- Economic. Manufacturing costs and profitability, cost of waste disposal, cost of energy and materials consumed; all normalised per product.

- Social. Yearly number of work injuries (divided into short-medium and long term) or fatalities, employees turnover rate, proportion of permanent employees, time available on education and training seminars offered by the firm to its personnel.

\section{Foundry Energy Efficient Process Metrics}

Although resource efficiency covers only a part of the many decision making metrics outlined above, it is of great importance for the foundry industry because of its energy intensive nature. The authors have already proposed in a previous work a correction to the conventional metrics - proposing a new, original set - to better evaluate and benchmark energy efficient metal casting [27]. This section summarises the conventional and new set of metrics and adds some additional information necessary to present the case study.

Two different approaches can be identified in evaluating the usual way to assess the performance of energy consumption in manufacturing plants (and, thus, in foundries). A "bottom-up" methodology based on first principles and a relatively accurate analysis of sub-processes and a "top-down" assessment based on aggregate data in terms of time (e.g. yearly data) and space (e.g. overall measurements made at the boundary of the factory system) [28].

As part of the second category, the calculation of the Specific Energy Consumption (SEC) has been particularly successful (although sometimes called and calculated in slightly different ways) both in the industry and academic research thanks to the minimal amount of input data required [1,28-34]. One of the most popular expressions of SEC is obtained dividing the total energy input of the plant $E_{i n, t o t}$ (including also all the ancillary processes, e.g. machining and heat treatment) by the total mass of products shipped to the customer $m_{s}$ within a certain representative amount of time (usually one year). Alternatively, some authors (e.g. Arasu and Jeffrey [33]) consider the amount of metal melted $m_{m}$ in place of $m_{s}$. Unfortunately, both quantities are usually called generically SEC creating the risk to mix and wrongly compare their different values. Thus, two quantities, $S E C_{o}$ and $S E C_{m}$, are defined:

$$
S E C_{o}=\frac{E_{\text {in, tot }}}{m_{s}}
$$




$$
S E C_{m}=\frac{E_{\text {in }, t o t}}{m_{m}}
$$

Both metrics measure the overall performance of the foundry.

Furthermore, it is not recommended to compare significantly different types of alloys (e.g. ferrous versus aluminium-based) or processes (e.g. sand casting versus investment casting) on the basis of SEC. This reason is twofold. Firstly, the metric does not contain any information about the thermodynamic properties of the alloy that influence the energy input of the melting phase (the main contributor to the total [1]). Secondly, significantly different casting practices would differ too much in terms of sub-processes (with their relevant energy inputs) and type of product to allow a reasonable comparison.

Another popular indicator usually considered to analyse the performance of foundries is yield. This is a measure of material efficiency (that indirectly controls also energy efficiency [35]) defined as the ratio of the material's mass at the boundaries of the process considered (i.e. before and after it). Also in this case the term is often used in an ambiguous way without clarifying where the mentioned boundaries are considered for the calculation (e.g. overall yield versus mould yield). The authors have decided to resolve this problem considering the parameter Operational Material Efficiency (OME) that describes unambiguously the material efficiency across the full factory process in a similar way to what SEC does for energy efficiency:

$$
O M E=\frac{m_{s}}{m_{m}}
$$

In a previous work by the authors, a correction to the expression of SEC was proposed following a more rigorous definition of efficiency as a non-dimensional parameter - called $\eta_{o}$ - that includes also some thermodynamic properties of the alloy being processed [27]:

$$
\eta_{o}=\frac{m_{S} \Delta h_{l}}{E_{\text {in,tot }}}=\frac{\Delta h_{l}}{S E C_{o}}
$$

where $\Delta h_{l}$ is the specific enthalpy rise to the liquidus point of the metal alloy. The expression of $\eta_{o}$ is derived comparing the actual total energy input in the foundry to the ideal minimum required to change the phase of the alloy (with no material losses across the full chain of processes). Although this ideal reference case can be seen as a very restrictive condition to consider, it is a clearly defined state that is valid for any type of shape casting process and provides a benchmarking value within a "universal" scale bounded between 0 and 1 .

Considering the importance of the melting phase as a proportion of the overall energy balance, a dedicated melting efficiency $\eta_{m}$ was proposed (and defined similarly to $\left.\eta_{o}\right)$ :

$$
\eta_{m}=\frac{m_{m} \Delta h_{s h}}{E_{\text {in, } m}}=\frac{\Delta h_{s h}}{O M E S E C_{m}}
$$

where $E_{i n, m}$ is the melting energy input and $\Delta h_{s h}$ is the specific enthalpy rise to the superheat temperature of the metal alloy. The superheat temperature is located beyond 
the liquidus point where the charge has to be brought to guarantee a satisfactory pouring into the mould (e.g. the correct level of fluidity with no risks of "cold runs" [36]). The choice of the superheat temperature as a reference point to define $\eta_{m}$ (in contrast to the liquidus temperature for $\eta_{o}$ ) is justified observing that it is an "external constraint" for the melting phase, dictated by the subsequent pouring.

The proposed new set of metrics is completed, by (optional) energy fractions $\varphi_{i}$ (for each step of the process) defined as

$$
\varphi_{i}=\frac{E_{i n, i}}{E_{i n, t o t}}
$$

where $E_{i n, i}$ is the generic $i$-th phase energy input. In particular, the energy fraction of melting $\varphi_{m}$ is important and cannot be considered optional like the remaining $\varphi_{i}$ to complete a minimal assessment.

The results shown in the next section required a slight tweaking to the methodology presented so far. Since the melting energy input was not available in isolation, the aggregate value of melting and holding was used to represent all quantities with subscript " $m$ ". As a consequence, no material loss (actually accounting for a few percentage points) was assumed between melting and holding when computing $S E C_{m}$. All other quantities are unaffected and it will be shown that this minor approximation will not influence the conclusions.

Finally, this new set of metrics could be integrated in a system analysis tools to visualise material and energy flows like the one developed by Pagone et al. [37,38].

\section{Case study: transfer case for a car}

Three different non-ferrous materials are considered to manufacture a car transfer case with a High Pressure Die Casting (HPDC) process [36]:

- Aluminium alloy A380 processed in a cold chamber machine with data obtained from an industrial contact. Monthly energy consumption meter readings were added up to obtain the total annual energy consumption. The data from two years of production have been combined to obtain the relevant average figure.

- Magnesium alloy AZ91D processed in a cold chamber machine [39] with data available in the scientific literature [1]. Overall energy consumption data is reported on annual basis for two years. Also in this case, an average value has been calculated.

- Zinc alloy ZA-8 processed in a hot chamber machine with data available in the scientific literature $[1,40]$. Monthly total energy consumption values are combined to calculate the annual figure available, in this case, only for one year.

The cumulative data of energy consumption include also the production of other automotive parts but the relevant error introduced it is expected to be consistent with the limits of approximation of this analysis. Mechanical (Table 1) and thermophysical properties of the materials have been calculated based on the data available on the online database MatWeb [41]. 
Table 1. Mechanical properties of three alloys used for the production of car transfer cases. Data: MatWeb [41]

\begin{tabular}{lccc}
\hline Quantity & Al-A380 & Mg-AZ91D & Zn-ZA8 \\
\hline Tensile strength (MPa) & 324 & 230 & 374 \\
Yield strength (MPa) & 159 & 150 & 290 \\
Elongation to break (\%) & 3.5 & 3 & 8 \\
Density (g/cm ${ }^{3}$ ) & 2.76 & 1.81 & 6.3 \\
Modulus of elasticity (GPa) & 71 & 45 & 85.5 \\
\hline
\end{tabular}

\subsection{Results}

A comparison of traditional metrics shows that the material efficiency $O M E$ is reasonably similar in all cases and energy efficiency appears to be quite similar between the aluminium and zinc alloys, whereas the magnesium alloy performs more poorly (Fig. 2). In this context, the proposed metric $\varphi_{m h}$ appears useful in identifying that the lower performance of the magnesium alloy is caused by the significantly higher proportion of energy spent in other process steps beyond melting and holding.

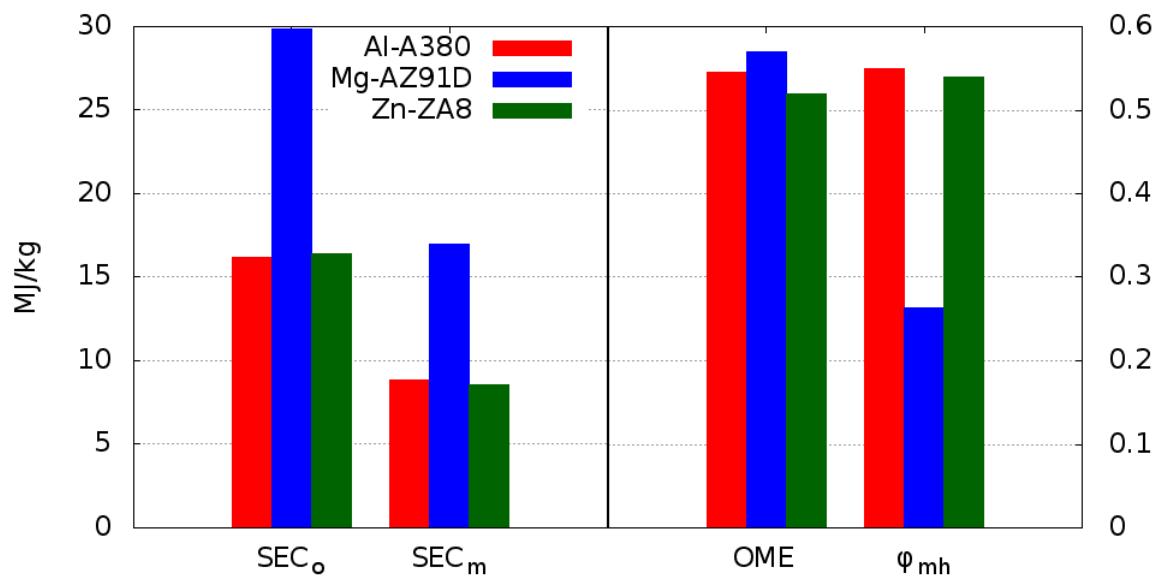

Fig. 2. Comparison of the traditional energy and material efficiency metrics for three alloys used for the production of car transfer cases. SEC: Specific Energy Consumption; OME: Operational Material Efficiency; $\varphi_{m h}$ : sum of melting and holding fractions of the total energy input.

However, more interesting observations can be made comparing SEC to $\eta_{o}$ (Fig. 3). To make this comparison simpler, the inverse of SEC - SEC ${ }^{-1}-$ is represented, i.e. it is plotted how many kilograms of product can be manufactured for every gigajoule of energy spent. In this way, larger values of $S E C^{-1}$ and the efficiencies represent con- 
sistently more energy efficient processes. Although SEC is fairly similar for both the aluminium and the zinc alloys, when considering the thermodynamic characteristics of the materials through $\eta_{o}$, a significant difference becomes visible. Specifically, the process based on ZA-8 appears less efficient, suggesting that it may be possible to improve the casting practice to reach a higher energy efficiency. Furthermore, comparing the values of $\eta_{m}$, the plant processing the zinc alloy exhibits a clearly lower efficiency in the melting and holding phase. Thus, the proposed metrics have identified a potential opportunity to improve the process and reduce the energy consumption to produce the transfer case.

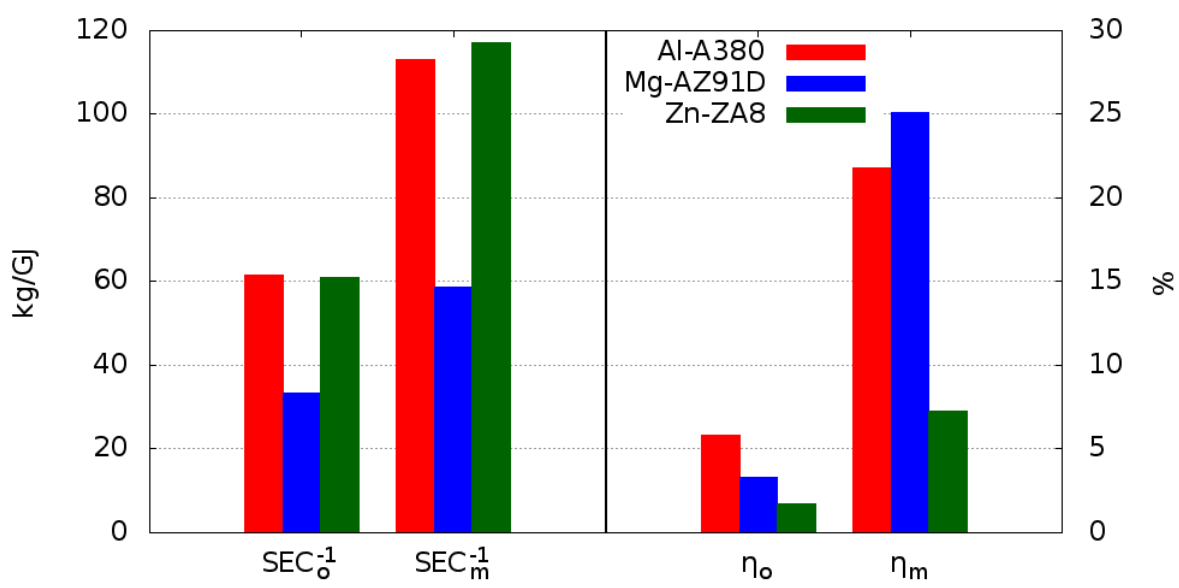

Fig. 3. Comparison of the Specific Energy Consumption (SEC) - through its inverse function $\mathrm{SEC}^{-1}$ - with the proposed energy efficient metrics $\eta_{o}$ and $\eta_{m}$ for three alloys used for the production of car transfer cases.

\subsection{Discussion}

The following limitations should be considered.

- The effect of weather (in general) and of the yearly average temperature (in particular) has not been included and may have a non-negligible effect on the energy efficient metrics [1].

- Considerations on the cost of the materials should be carried out and may have a significant impact on the conclusions. However, this is considered an aspect outside the scope of this work.

Thanks to the analysis based on the proposed set of metrics, it has been possible to identify that:

- the lower energy efficiency of the process based on the AZ91D alloy is caused by the significant impact of other process steps beyond melting and holding; 
- although the process based on the AZ91D alloy is the least efficient, its melting and holding phases appear to be relatively efficient;

- the process based on the ZA-8 alloy has the potential to improve significantly its overall efficiency because the efficiency of the melting and holding phases show a non-negligible margin of improvement.

Concerning the last observation, the yearly volume of production of the plant producing zinc alloy parts is significantly lower than the one of the plant based on the aluminium alloy and larger production rates permit improvements in the energy efficiency of foundries [28,34].

Therefore, it may be concluded that the process based on the ZA-8 alloy has the potential to be the most energy efficient and, thus, the one to prefer among the three considered. On the other hand, the density of the ZA-8 alloy is more than twice the one of its closest competitor (i.e. A380) although its remaining mechanical properties are clearly superior (Table 1). Taking advantage of such properties it would be possible to redesign the transfer case using a lower amount of material that could somewhat mitigate the considerable difference in weight. However, this difference would likely remain significant and it may happen that a potential reduction in energy consumption during manufacturing can be offset, for example, by a poorer fuel economy of the vehicle (i.e. an increase of the energy consumed) during the use phase.

Thus, only a more comprehensive Life Cycle Assessment (LCA) of the product may determine the most energy efficient material. However, also this study is outside of the scope of this work.

\section{Conclusion}

After a short introduction describing the importance of metal casting for the automotive sector, a summary of decision making metrics in manufacturing systems has been presented with its categorisation in five different classes. Alongside the traditional parameters in the domain of cost, time, quality and flexibility, sustainability indicators (that have not reached their full maturity, yet) have been broadly described by means of examples and the tools used for their assessment.

A focus on the quantification of energy efficiency in metal casting foundries presented traditional metrics widely adopted in the industrial and academic research. A correction to these metrics to improve their ability to capture more information about the process was proposed based on the thermodynamic properties of the alloy being cast.

Finally, a case study comparing the energy efficiency of a high pressure die casting process to produce a transfer case for a car with three different non-ferrous alloys (i.e. aluminium A380, magnesium AZ91D and zinc ZA-8) has been used to illustrate the potential of the new set of metrics proposed. Using this set of metrics, additional details about the processes were identified with, in particular, the potential to improve the efficiency of the plant based on the ZA-8 alloy.

Although it can be stated with reasonable confidence that a transfer case produced in zinc alloy may be the best option to minimise the manufacturing energy consump- 
tion within the considered options, several other aspects (e.g. LCA analysis, overall cost, design requirements for the full car, etc.) in a multi-disciplinary context should be considered to provide a final answer.

The new set of metrics proposed appears as a valuable resource for the analysis of metal casting manufacturing processes, as long as similar products with similar practices are considered.

\section{Acknowledgements}

The authors would like to acknowledge the UK EPSRC projects "Small is Beautiful" (EP/ M013863/1) and "Energy Resilient Manufacturing 2: Small is Beautiful Phase 2 (SIB2)" (EP/P012272/1), comprising the Energy Resilient Manufacturing portfolio, for funding this work.

\section{References}

1. Eppich, R.E.: Energy Use in Selected Metalcasting Facilities. US Department of Energy, Washington, D.C. (2004).

2. The Global Market for Aluminum Castings to 2021, http://www.metalbulletinstore.com/, last accessed 2018/03/02.

3. Brief Profile of Indian Foundry Industry, http://www.foundryinfoindia.org/profile_of_indian.aspx, last accessed 2018/03/02.

4. Chryssolouris, G.: Manufacturing Systems: Theory and Practice. $2^{\text {nd }}$ edn. Springer-Verlag, New York (2006).

5. Pagone, E., Salonitis, K., Jolly, M.: Energy Efficient Casting Processes. In: Gupta, K. (eds.) Innovation in Manufacturing for Sustainability, in press, Springer (2018).

6. Fisseler, D., Schneider, A., Lourenço, E.J., Baptista, A.J.: Combining Process Based Monitoring with Multi-layer Stream Mapping. In: Campana, G., Howlett, R.J., Setchi, R., Cimatti, B. (eds.) Selected Papers on Sustainable Design and Manufacturing, pp. 559-568. Springer, Cham (2017).

7. Baptista, A.J., Lourenço, E.J., Silva, E.J., Estrela, M.A., Peças, P.: Integration of EcoEfficiency and Efficiency Assessment Methodologies: The Efficiency Framework. In: Campana, G., Howlett, R.J., Setchi, R., Cimatti, B. (eds.) Selected Papers on Sustainable Design and Manufacturing, pp. 613-623. Springer, Cham (2017).

8. Ferrera, E., Rossini, R., Baptista, A.J., Evans, S., Hovest, G.G., Holgado, M., Lezak, E., Lourenço, E.J., Masluszczak, Z., Schneider, A., Silva, E.J., Werner-Kytölä, O., Estrela, M.A.: Toward Industry 4.0: Efficient and Sustainable Manufacturing Leveraging MAESTRI Total Efficiency Framework. In: Campana, G., Howlett, R.J., Setchi, R., Cimatti, B. (eds.) Selected Papers on Sustainable Design and Manufacturing, pp. 624-633. Springer, Cham (2017).

9. Elkington, J.: The Triple Bottom Line of 21st Century Business. Capstone, Mankato (1997).

10. United Nations General Assembly: Report of the World Commission on Environment and Development: Our Common future. Oxford University Press, Oxford (1987).

11. Li, Y., Holgado, M., Benedetti, M., Evans, S.: Conceptual Analysis of Eco-Efficiency and Industrial Symbiosis: Insights from Process Industry. In: Campana, G., Howlett, R.J., 
Setchi, R., Cimatti, B. (eds.) Selected Papers on Sustainable Design and Manufacturing, pp. 601-612. Springer, Cham (2017).

12. Djokic, M.R., Van Geem, K.M., Heynderickx, G.J., Dekeukeleire, S., Vangaever, S., Battin-Leclerc, F., Bellos, G., Buysschaert, W., Cuenot, B., Faravelli, T., Henneke, M., Jakobi, D., Lenain, P., Munoz, A., Olver, J., Van Goethem, M., Oud, P.: IMPROOF: Integrated Model Guided Process Optimisation of Steam Cracking Furnaces. In: Campana, G., Howlett, R.J., Setchi, R., Cimatti, B. (eds.) Selected Papers on Sustainable Design and Manufacturing, pp. 589-600. Springer, Cham (2017).

13. Birolini, A.: Reliability Engineering: Theory and Practice. 7th edn. Springer, Berlin (2013).

14. Taguchi, G, Elsayed, E.A., Hsiang, T.: Quality Engineering in Production Systems. McGraw-Hill, New York (1989).

15. Browne, J., Rathmill, K., Sethi, S.P., Stecke, K.E.: Classification of Flexible Manufacturing Systems. In: The FMS Magazine, pp. 114-117 (1984).

16. Buzacott, J.A.: The Fundamental Principles of Flexibility in Flexible Manufacturing Systems. In: Proceedings of the First International Conference on Flexible Manufacturing Systems, pp. 13-22. Amsterdam (1982).

17. Falkner, C.H.: Flexibility in Manufacturing Plants. In: Stecke, K.E., Suri, R. (eds.) Proceedings of the Second ORSA/TIMS Conference of Flexible Manufacturing Systems: Operations Research Models and Applications, pp. 95-106 (1986).

18. Kumar, V.: Entropic Measures of Manufacturing Flexibility. International Journal of Production Research, 25(7), 957-966 (1987).

19. Brill, P.H., Mandelbaum, M.: On Measures of Flexibility in Manufacturing Systems. International Journal of Production Research, 27(5), 747-756 (1989).

20. Makino, H.: Versatility Index - An Indicator for Assembly System Selection. Annals of the CIRP, 39(1), 15-18 (1990).

21. Salonitis, K., Stavropoulos, P.: On the Integration of the CAx Systems Towards Sustainable Production. Procedia CIRP 9, 115-120 (2013).

22. Chen, D., Heyer, S., Ibbotson, S., Salonitis, K., Steingrímsson, J.G., Thiede, S.: Direct Digital Manufacturing: Definition, Evolution, and Sustainability Implications. Journal of Cleaner Production 107, 615-625 (2015).

23. Chen, D., Schudeleit, T., Posselt, G., Thiede, S.: A State-of-the-Art Review and Evaluation of Tools for Factory Sustainability Assessment. Procedia CIPR 9, 85-90 (2013).

24. Sustainable development in the European Union: Monitoring Report on Progress Towards the Sustainable Development Goals in an European Union Context, http://ec.europa.eu/eurostat/documents/3217494/8461633/KS-04-17-780-EN-N.pdf, last accessed 2018/03/02.

25. The Sustainability Metrics: Sustainable Development Progress Metrics, http://nbis.org/nbisresources/metrics/triple_bottom_line_indicators_process_industries.pdf, last accessed 2018/03/02.

26. Danis, A.: Barometer of Sustainability. In: Developing Ideas 9, p. 1. International Institute for Sustainable Development, New York (1997).

27. Pagone, E., Salonitis, K., Jolly, M.: Energy and Material Efficiency Metrics in Foundries. Procedia CIRP 21, 421-428 (2018).

28. Brevick, J.: Energy Consumption of Die Casting Operations. US Department of Energy, Washington, D.C. (2004).

29. Schifo, J.F., Radia, J.T.: Theoretical/Best Practice Energy Use in Metalcasting Operations. US Department of Energy, Washington, D.C. (2004). 
30. Arens, M., Schleich, J., Worrell, E.: Energy Efficiency Improvement in the German Steel Sector - more than Window Dressing? ECEEE 2012, Industrial Summer Study, (2012).

31. Phylipsen, G.J.M., Blok, K., Worrell, E.: Handbook on International Comparisons of Energy Efficiency in the Manufacturing Industry. Utrecht University, Utrecht (1998).

32. Lazzarin, R.M., Noro, M.: Energy Efficiency Opportunities in the Production Process of Cast Iron Foundries: an Experience in Italy. Applied Thermal Engineering 90, 509-520 (2015).

33. Arasu, M., Jeffrey, R.L.: Energy Consumption Studies in Cast Iron Foundries. In: Transaction of the 57th IFC (2009).

34. Heinemann, T.: Energy and Resource Efficiency in Aluminium Die Casting. Springer, Switzerland (2016).

35. Salonitis, K., Jolly, M., Zeng, B, Mehrabi, H.: Improvements in Energy Consumption and Environmental Impact by Novel Single Shot Melting Process for Casting. Journal of Cleaner Production 137, 1532-1542 (2016).

36. Jolly, M.: Castings. In: Milne, I., Ritchie, R.O., Karihaloo, B. (eds.) Comprehensive Structural Integrity, pp. 377-466. Elsevier, Oxford (2003)

37. Pagone, E., Jolly, M., Salonitis, K.: The Development of a Tool to Promote Sustainability in Casting Processes. Procedia CIPR 55, 53-58 (2016).

38. Pagone, E., Jolly, M., Salonitis, K.: A Tool to Promote Sustainability in Casting Processes: Development Highlights. In: Campana, G., Howlett, R.J., Setchi, R., Cimatti, B. (eds.) Selected Papers on Sustainable Design and Manufacturing, pp. 172-184. Springer, Cham (2017).

39. Luo, A.A.: Magnesium Casting Technology for Structural Applications. Journal of Magnesium and Alloys 1, 2-22 (2013).

40. Yakoub, M.M.: Squeeze Casting of Zinc-Aluminium (ZA) Alloys and ZA-27/SIC Composites. Doctoral thesis, Loughborough University (1987).

41. MatWeb, Your Source for Materials Information, http://www.matweb.com/, last accessed 2018/03/02. 


\title{
Metal casting energy efficient metrics for material selection of automotive parts
}

\author{
Pagone, Emanuele
}

Springer

Pagone E, Papanikolaou M, Salonitis K, Jolly M. (2019) Metal casting energy efficient metrics for material selection of automotive parts. In: Dao D., et al., (Eds), Sustainable Design and Manufacturing 2018. KES-SDM 2018. Smart Innovation, Systems and Technologies, Volume 130, pp. 290-303

https://doi.org/10.1007/978-3-030-04290-5_29

Downloaded from Cranfield Library Services E-Repository 